\title{
Manifestaciones Orales de la Infección por COVID-19
}

\author{
Oral Manifestations of COVID-19
}

\author{
María Eugenia Nemeth Kohanszky1; Carolina Paz Matus Abásolo² \& Rolando Rafael Carrasco Soto ${ }^{3,4}$
}

\begin{abstract}
NEMETH, K. M. E.; MATUS, A. C. P. \& CARRASCO, S. R. R. Manifestaciones orales de la infección por COVID-19. Int. J. Odontostomat., 14(4):555-560, 2020.

RESUMEN: El 8 de enero de 2020, el Centro Chino para el Control y Prevención de Enfermedades anunció oficialmente la identificación de una nueva cepa de coronavirus (SARS-CoV-2) como el patógeno causante de la pandemia mundial de COVID-19. Las principales manifestaciones clínicas producidas por SARS-CoV-2 se encuentran ampliamente descritas en la literatura, sin embargo, la información acerca de las alteraciones que podrían producirse a nivel oral, es escasa. Se ha sugerido que la cavidad oral es un perfecto hábitat para la invasión por SARS-CoV-2 debido a la especial afinidad que tiene el virus por células con los receptores para la enzima convertidora de angiotensina (ECA2) como son las del tracto respiratorio, mucosa oral, lengua y glándulas salivales, pudiendo afectar, de este modo, el funcionamiento de las glándulas salivales, las sensaciones del gusto, olfato y la integridad de la mucosa oral. El nuevo coronavirus tendría la capacidad de alterar el equilibrio de la microbiota oral, lo que sumado a un sistema inmune deprimido permitiría la colonización por infecciones oportunista. Se ha establecido que un correcto higiene oral podría disminuir la incidencia y gravedad de las principales complicaciones del COVID-19. El objetivo de este estudio es realizar una revisión y análisis de la evidencia disponible en relación a las manifestaciones orales a nivel de las mucosas, trastornos de las glándulas salivales y alteraciones en el sistema olfatorio y gustativo en el contexto de la infección por SARS-CoV-2.
\end{abstract}

PALABRAS CLAVE: COVID-19, SARS-CoV-2, manifestaciones orales

\section{INTRODUCCIÓN}

El 8 de enero de 2020, el Centro Chino para el Control y Prevención de Enfermedades anunció oficialmente la identificación de una nueva cepa de coronavirus (SARS-CoV-2) como el patógeno causante de la pandemia mundial de COVID-19 (Li et al. 2020). En comparación con otras pandemias recientes el COVID-19 tiene, en general, una presentación clínica menos severa, sin embargo, se propaga con mayor facilidad, lo que ha generado aproximadamente 16,7 millones de contagios y más de 650.000 muertes a nivel mundial, al 29 de julio del 2020 (World Health Organization, 2020).

La información disponible acerca de las principales manifestaciones clínicas producidas por SARSCoV-2 tales como fiebre, tos seca, diarrea y dificultad respiratoria es amplia y detallada, sin embargo, aquella sobre los síntomas reportados más recientemente, pre- sentes en su mayoría en una etapa más temprana de la infección, tales como lesiones cutáneas, alteraciones del olfato y repercusiones a nivel oral, es escasa.

El impacto del COVID-19 en la salud oral está principalmente determinado por el sistema inmunológico del paciente, la farmacoterapia que recibe y por la patogenia del virus. Se ha sugerido que la cavidad oral es un perfecto hábitat para la invasión por SARS-CoV-2 debido a la especial afinidad que tiene el virus por células con los receptores para la enzima convertidora de angiotensina (ECA2) como son las del tracto respiratorio, mucosa oral, lengua y glándulas salivales (Xu et al., 2020; Peng et al., 2020).

Se ha demostrado que el SARS-CoV-2 es un virus neurotrópico y mucotrópico, pudiendo afectar el funcionamiento de las glándulas salivales, las sensa-

\footnotetext{
${ }^{1}$ Cirujana Dentista, Facultad de Odontología, Universidad del Desarrollo, Concepción.

${ }^{2}$ Cirujana Dentista, Facultad de Odontología, Universidad de Chile.

${ }^{3}$ Especialista en Cirugía y traumatología Maxilofacial, Hospital San Juan de Dios, Santiago, Chile

${ }^{4}$ Especialista en Cirugía y traumatología Maxilofacial, Clínica Las Condes, Santiago, Chile.
} 
ciones del gusto, olfato y la integridad de la mucosa oral (Lechien et al., 2020). Estudios indicarían que este nuevo coronavirus posee la capacidad de alterar el equilibrio de la microbiota oral, lo que combinado con un sistema inmune deprimido permitiría la colonización por infecciones oportunistas (Dziedzic \& Wojtyczka, 2020). En la actualidad no se ha descubierto un fármaco eficiente y seguro contra el COVID19 y los potenciales incluyen reacciones adversas tales como trastornos hepáticos, renales, hematológicos, psiquiátricos, arritmias cardiacas, perforaciones intestinales e incluso lesiones a nivel oral (Agencia Española de Medicamentos y Productos Sanitarios, 2020; Amorim dos Santos et al., 2020; Godinho et al., 2020; de Melo Filho et al., 2012)

Actualmente, existen dudas acerca de si el origen de estas manifestaciones orales es resultado de la infección viral directa, si son producto del compromiso sistémico del paciente o si se presentan como reacciones adversas a los tratamientos recibidos para tratar el COVID-19.

En esta revisión se presenta la evidencia actual disponible en relación a las manifestaciones orales a nivel de las mucosas, trastornos de las glándulas salivales y alteraciones en el sistema olfatorio y gustativo en el contexto de la infección por SARSCoV-2.

\section{COVID-19 Y SALUD ORAL}

Existen múltiples artículos científicos que han investigado acerca de la cavidad oral como la principal vía de infección por SARS-CoV-2, las implicancias del alto riesgo de contagio en la práctica odontológica y el posible uso de la saliva para su diagnóstico (Melián et al., 2020; Pedrosa et al., 2020; Baghizadeh Fini, 2020). Sin embargo, son escasos los estudios que relacionan el COVID-19 con los trastornos de las glándulas salivales, alteraciones de gusto y olfato, lesiones intraorales e higiene oral, información que puede resultar de gran utilidad para la prevención, diagnóstico y tratamiento de la patología.

Trastornos de las glándulas salivales. La saliva posee una función vital para mantener la integridad de los tejidos de la cavidad oral dado que permite su lubricación, amortigua cambios de $\mathrm{pH}$ y posee una acción antibacteriana, antiviral y antifúngica. El volumen de secreción salival, en condiciones normales, depende de múltiples factores tales como temperatura, ritmo circadiano, tipo de sabor y estimulación quimiosensorial, masticatoria o táctil (Suzuki \& Iwata, 2018). En ocasiones, asociado principalmente a la condición sistémica del paciente y al consumo de ciertos fármacos, se desarrolla una hiposalivación. Esta se relaciona con la presencia de lesiones ulcerosas de la mucosas intraorales, disgeusia, disfagia y mayor susceptibilidad a infecciones (Aitken-Saavedra et al., 2017).

La presencia de hiposalivación y xerostomía ha sido reportada en infectados con SARS-CoV-2 (Pedrosa et al.). Estudios indican que pacientes con hiposalivación poseen un mayor riesgo de desarrollar una infección respiratoria severa, dado que al existir una menor secreción salival es posible que se altere la función de barrera de la mucosa de la vía aérea respiratoria, favoreciendo la adhesión y colonización viral. A su vez, la hiposalivación se relaciona con una disminución en proteínas salivales como mucinas, lisozima, catelicidina, lactoferrina, peroxidasa, aglutinina salival, alfa-defensinas, beta-defensinas y cistatinas, quienes podrían potencialmente impedir la replicación del virus, especialmente del SARS-CoV-2 (Baghizadeh Fini). Es por esto, que la hiposalivación puede ser considerada como un factor de riesgo para el desarrollo de infecciones respiratorias tales como el COVID-19.

Trastornos del gusto y olfato. El cuerpo humano utiliza tres modalidades sensoriales: olfato, gusto y quimioestesis. El sistema olfativo detecta sustancias químicas volátiles a través de neuronas sensoriales olfativas de la cavidad nasal y olores provenientes de alimentos por medio de la nasofaringe (olfato retronasal). El sistema gustativo responde a compuestos en la boca que provocan sensaciones de dulce, salado, amargo, agrio y umami. La quimioestesis detecta otros químicos, encontrados en hierbas o especias, que evocan sensaciones como ardor, enfriamiento u hormigueo. A menudo, estas modalidades se combinan y transfieren una experiencia única de sabor durante la comida, por ello es frecuente que, al existir una pérdida del olfato retronasal y alteración en el sistema somatosensorial, que transmite quimioestesis, se informe como una pérdida de gusto (Parma et al., 2020). Las alteraciones quimiosensoriales de estos sistemas pueden resultar en cambios del gusto y olfato cuantitativos (anosmia, hiposmia; ageusia, hipogeusia) y cualitativos (disgeusia, parosmia) respectivamente (Sepúlveda et al., 2020).

Recientemente, se ha reportado que el COVID19 se relacionaría con alteraciones del olfato $(A O)$ y/o 
del gusto (AG). La asociación de infecciones virales con $A O$ y $A G$ es frecuente ya que los virus pueden provocar una inflamación de la mucosa nasal y rinorrea. Sin embargo, el caso del COVID-19 parece ser algo particular, ya que no está asociado a estas características. Si bien se sabe que el SARS-CoV-2, por su afinidad con los receptores ECA2, puede infectar los queratinocitos de la lengua, no está del todo claro el mecanismo a través del cual afecta los sentidos y si bien una $A O$ puede conllevar a una $A G$ es posible que no se presenten en conjunto ya que poseen distintos mecanismos neurales periféricos y centrales (Parma et al.). Se cree que el virus puede tener la capacidad de infectar las células receptoras del gusto, los nervios craneales que portan el sabor y la información quimioestésica o que incluso podría infectar los vasos sanguíneos circundantes y las células del sistema nervioso central (Brann et al., 2020).

Estudios indican que la prevalencia de AO y AG para pacientes con COVID-19 varía entre un 58-86 \% y 54-88 \% respectivamente (Bénézit et al., 2020; Costa et al., 2020; Lechien et al.; Yan et al., 2020). De estas manifestaciones las más frecuentes seríanlas cualitativas, principalmente anosmia y ageusia (Lechien et al.). Se ha señalado que AO y AG le seguirían en prevalencia a la fiebre, tos seca y fatiga (Yan et al., 2020a,b; Menni et al., 2020) y otros autores incluso indican que serían las manifestaciones más prevalentes del COVID-19, sobre todo en pacientes que presenten una severidad de infección leve a moderada y que sean de sexo femenino (Lechien et al.). Por lo anterior, se los considera excelentes predictores de la infección y en caso de presentarlos se recomienda al paciente estar aislado hasta contar con los resultados de la prueba de laboratorio.

Hallazgos intraorales. En la cavidad oral se pueden presentar manifestaciones de enfermedades subyacentes de origen bacteriano o viral. Entre estas se destacan: úlceras orales, gingivorragia, glositis, halitosis y dolor orofacial. Las lesiones orales de etiología viral más prevalentes son las úlceras y lesiones ampollosas de los tejidos.

Diversos reportes de casos han confirmado la presencia de manifestaciones orales en pacientes con COVID-19 (Chaux-Bodard et al., 2020, de Maria et al., 2020, Lechien et al.; Carreras-Presas et al., 2020, Putra et al., 2020). Se cree que estas se deben principalmente al estado inmunocomprometido del paciente, a la mala higiene oral y la coinfección con otras infecciones virales o bacterianas (Pedrosa, et al.).
Los hallazgos intraorales más reportados en pacientes con COVID-19 corresponden a candidiasis y lesiones herpéticas. Se ha sugerido que las úlceras orales recurrentes podrían ser un síntoma inaugural del COVID-19. Sin embargo, como estos hallazgos aún son recientes en la literatura, no está claro si se deben a la infección por coronavirus propiamente tal o si son manifestaciones secundarias a la condición sistémica del paciente (Amorim dos Santos et al., 2020)

Candidiasis. Hasta ahora, no se ha prestado mayor atención a la prevalencia de infecciones fúngicas en pacientes con COVID-19, sin embargo, existe conocimiento acerca de la posibilidad de que estos pueden experimentar linfocitopenia, requerimiento de hospitalización en una unidad de cuidados intensivos, necesidad de antibióticos de amplio espectro, corticoides y presentar enfermedades subyacentes, las que sumadas, comprometerían severamente al sistema inmune, considerándose factores de riesgo para la coinfección con candidiasis orofaríngea (CO).

En más del $80 \%$ de los casos de $\mathrm{CO}$, la especie responsable sería Candida albicans y si bien esta forma parte de nuestra flora endógena, la infección se desarrolla cuando las defensas locales del huésped se debilitan, pudiendo presentarse alteraciones del gusto, glosodinia y disfagia. En caso de que la CO no sea tratada o el tratamiento sea ineficaz, la infección puede propagarse regionalmente desde la orofaringe al esófago o sistemáticamente a través del torrente sanguíneo o el tracto gastrointestinal superior, lo que podría producir una candidemia con morbilidad significativa o incluso mortalidad. Por lo tanto, la detección oportuna de $\mathrm{CO}$ y la identificación precisa de los agentes etiológicos en pacientes que padecen COVID-19 son importantes para aplicar una terapia efectiva (Amorim dos Santos et al.; Salehi et al., 2020).

Los estudios acerca de $\mathrm{CO}$ en pacientes con COVID-19 son escasos. Una investigación realizada a más de 1000 pacientes infectados por SARS-CoV2 reveló que el $5 \%$ de ellos presentó CO, en promedio 8 días posteriores al diagnóstico de COVID-19, siendo Candida albicans la responsable de un $71 \%$ de los casos y los pacientes mayores de 49 años un $80 \%$ de los afectados (Salehi et al.).

Teniendo en cuenta el curso clínico, la progresión y la gravedad del COVID-19, la mayoría de los pacientes con cuadros severos presentarían al me- 
nos uno de los factores de riesgo mencionados para el desarrollo de $\mathrm{CO}$, siendo especialmente vulnerables los adultos mayores.

Lesiones herpéticas. Carreras-Presas et al., reportaron 3 casos de manifestaciones orales probablemente asociadas a la infección por SARS-CoV-2, uno de los cuales pertenecía a un caso sospechoso, el segundo a un caso probable y el tercero a un caso confirmado de infección por COVID-19. Los 3 pacientes presentaron úlceras o ampollas a nivel oral, correspondientes a lesiones elementales, comúnmente observadas en otros procesos virales como fiebre aftosa, gingivoestomatitis herpética e infección oral por citomegalovirus. En los 2 primeros casos se encontraban en tejido queratinizado, como suele observarse en lesiones orales de herpes simple (Scully \& Samaranayake, 2016). En el tercer caso, si bien la manifestación oral tenía aspecto herpetiforme, esta se encontraba ubicada en tejido queratinizado y no queratinizado, siendo más compatible con un eritema multiforme (EM) (Trayes et al., 2019).

A su vez, en una carta al editor publicada en mayo 2020, se reportó la presencia de lesiones maculares y petequias a nivel oral en el paladar en cuatro pacientes hospitalizados con infección por COVID-19, como parte de un patrón de lesiones cutáneas tipo EM, siendo el virus herpes simple y Mycoplasma pneumoniae los principales agentes causales asociados a EM (Jimenez-Cauhe et al., 2020).

Posterior a esto, en junio 2020 en otra carta al editor se reportaron las características clínicas y microscópicas del caso de un paciente positivo para SARS-CoV-2 quien, además de los principales síntomas de fiebre, tos y dificultad respiratoria, presentó úlceras orales dolorosas y máculas eritematosas múltiples en paladar duro, lengua y labios, como parte del cuadro infeccioso que incluyó también lesiones cutáneas vesiculosas pequeñas, similares a petequias, de etiología desconocida. Los aspectos clínicos y microscópicos de las lesiones mostraron áreas de hemorragia y pequeños vasos trombóticos, sugiriendo que el SARS-CoV-2 puede causar lesiones orales, siendo estas una reacción primaria. Este reporte, es el único hasta la fecha que incluye el estudio histopatológico de las lesiones orales y que menciona un posible mecanismo etiopatogénico (Soares et al., 2020).

Control de la higiene oral. El vínculo entre un buen cuidado bucal y un menor riesgo de infecciones respiratorias virales agudas se ha establecido en varios estudios (Imsand et al., 2002; Quagliarello et al., 2005; Sampson et al., 2020). Se ha determinado que una higiene oral inadecuada puede aumentar el intercambio bacteriano entre pulmones y boca, lo que a su vez incrementaría el riesgo de infecciones pulmonares y la presencia de complicaciones bacterianas posteriores a la infección viral. Así mismo, se ha establecido una conexión entre presencia de enfermedad periodontal y complicaciones postvirales, dado que citoquinas como IL-1a, IL1b, TNF-a presentes en sacos periodontales pueden infiltrarse en la saliva a través del líquido crevicular gingival y ser aspirados, incrementándose el riesgo de inflamación o infección a nivel pulmonar (Sampson et al.).

Se ha determinado que existe una asociación entre el cuidado oral y la incidencia de neumonía en pacientes conectados a ventilador mecánico, especialmente en aquellos mayores de 60 años con comorbilidades (Sampson et al.). Investigaciones señalan que dentro de las principales complicaciones de los pacientes con COVID-19 se encuentran la neumonía y el síndrome de dificultad respiratoria aguda (SDRA), determinándose el diagnóstico de SDRA cuando los niveles de oxígeno caen y se requiere ventilación asistida. Esto es respaldado por el informe oficial italiano, en donde se indica que el $96,5 \%$ de las complicaciones derivadas de la infección por COVID-19 fueron SDRA, lo que sugiere que los pacientes tienen más probabilidad de morir por complicaciones de infección postviral que por COVID19 (European Centre for Disease Prevention and Control, 2020).

Si bien son necesarios más estudios para establecer la importancia de la higiene oral en el pronóstico del paciente COVID-19, se recomienda mantener o incluso mejorar la higiene oral durante la infección por SARS-CoV-2 para reducir la carga bacteriana y el riesgo potencial de una sobreinfección (Sampson et al.).

\section{CONCLUSIÓN}

Las principales manifestaciones orales por COVID-19 reportadas en la literatura son: hiposalivación, xerostomía, ageusia, hipogeusia, disgeusia, lesiones herpéticas y candidiasis. 
Se ha comprobado que la cavidad oral es el portal de ingreso perfecto para infección por SARSCoV-2 por la especial afinidad del virus con los receptores ECA2 presentes en las células de la mucosa oral, lengua y glándulas salivales. Una vez instaurada la enfermedad, el virus tendría la capacidad de alterar el equilibrio de la microbiota oral e inmunosuprimir al paciente, permitiendo la posible aparición de infecciones oportunistas. Esto, combinado con la terapia farmacológica y trastornos de las glándulas salivales, cuya etiología aún no está del todo clara, contribuiría al desarrollo de las manifestaciones orales y trastornos sensoriales, los cuales podrían presentarse en una etapa temprana y ser de gran utilidad para la identificación diagnóstica oportuna de esta patología.

Es de gran relevancia incorporar rutinariamente la evaluación de signos y síntomas orales en pacientes diagnosticados con infección por SARS-CoV-2 para determinar si las manifestaciones orales forman parte de la semiología de la infección o, si corresponden,a distintas entidades patológicas, las que se ven favorecidas por la respuesta inmune que desencadena este nuevo coronavirus, así como también incentivar una adecuada higiene oral para disminuir el riesgo de complicaciones como neumonía y SDRA.

NEMETH, K. M. E.; MATUS, A. C. P. \& CARRASCO, S. R. R. Oral manifestations of COVID-19. Int. J. Odontostomat., 14(4):555-560, 2020.

ABSTRACT: On January 8, 2020, the Chinese Center for Disease Control and Prevention officially reported the identification of a new strain of coronavirus (SARS-CoV-2) as the pathogen causing the global COVID-19 pandemic. The main clinical manifestations produced by SARS-CoV-2 are well described in the literature. However, the information about oral manifestations is limited. It has been suggested that the oral cavity is a perfect habitat for SARS-CoV-2 invasion due to the special affinity that the virus has for cells with the receptors for the converted angiotensin enzyme (ACE2), present in the respiratory tract, oral mucosal, tongue and salivary glands, thus being able to affect the functioning of the salivary glands, sensations of taste, smell and integrity of the oral mucosa. The new coronavirus could have the ability to alter the balance of the oral microbiota, which added to a depressed immune system would allow opportunistic infections colonization. It has been established that correct oral hygiene could decrease the incidence and severity of the main complications of COVID-19. The objective of this study is to review and analyze the available evidence in relation to oral manifestations at the mucosa, salivary glands disorders and olfactory and gustatory alterations in the context of SARS-CoV-2 infection.

KEYWORDS: COVID-19, SARS-CoV-2, oral manifestations

\section{REFERENCIAS BIBLIOGRÁFICAS}

Agencia Española de Medicamentos y Productos Sanitarios (AEMPS). Sospechas de Reacciones Adversas Notificadas con Tratamientos Utilizados en COVID-19. Madrid, Ministerio de Sanidad, Gobierno de España, 2020. Disponible en: https:// www.aemps.gob.es/la-aemps/ultima-informacion-de-la-aempsacerca-del-covid\%E2\%80\%9119/sospechas-de-reacciones-adversas-notificadas-con-tratamientos-utilizados-en-covid-19/

Aitken-Saavedra, J. P.; Olid, C.; Escobar, A.; Parry, Y.; Duarte da Silva, K. \& Morales-Bozo, I. Características salivales y estado sistémico de sujetos con xerostomía. Rev. Clin. Periodoncia Implantol. Rehabil. Oral, 10(2):118-20, 2017.

Amorim dos Santos, J. A.; Normando, A. G. C.; Carvalho da Silva, R. L.; Monteiro De Paula, R.; Cembranel, A. C.; Santos-Silva, A. R. \& Silva Guerra, E. N. Oral mucosal lesions in a COVID-19 patient: New signs or secondary manifestations? Int. J. Infect. Dis., 97:326-8, 2020.

Baghizadeh Fini, M. Oral saliva and COVID-19. Oral Oncol., 108:104821, 2020.

Bénézit, F.; Le Turnier, P.; Declerck, C.; Paillé, C.; Revest, M.; Dubée, V.; Tattevin, P. \& RAN COVID Study Group. Utility of hyposmia and hypogeusia for the diagnosis of COVID-19. Lancet Infect. Dis., 2020. DOI: https://www.doi.org/10.1016/S14733099(20)30297-8

Brann, D. H.; Tsukahara, T.; Weinreb, C.; Lipovsek, M.; Van den Berge, K.; Gong, B.; Chance, R.; Macaulay, I. C.; Chou, H.; Fletcher, R.; et al. Non-neuronal expression of SARS-CoV-2 entry genes in the olfactory system suggests mechanisms underlying COVID-19-associated anosmia. bioRxiv, 2020. DOI: https:// www.doi.org/10.1101/2020.03.25.009084

Carreras-Presas, C. M.; Amaro Sánchez, J.; López-Sánchez, A. F.; Jané-Salas, E. \& Somacarrera Pérez, M. L. Oral vesiculobullous lesions associated with SARS-CoV-2 infection. Oral Dis., 2020. DOI: https://www.doi.org/10.1111/odi.13382

Chaux-Bodard, A. G.; Deneuve, S. \& Desoutter, A. Oral manifestation of Covid-19 as an inaugural symptom? J. Oral Med. Oral Surg., 26:18, 2020.

da Costa, K. V. T.; Carnaúba, A. T. L.; Rocha, K. W.; de Andrade, K. C. L.; Ferreira, S. M. S. \& Menezes, P. L. Olfactory and taste disorders in COVID-19: a systematic review. Braz. J. Otorhinolaryngol., 2020. DOI: https://www.doi.org/10.1016/ j.bjorl.2020.05.008

de Melo Filho, M. R.; da Silva. C. A. D.; da Rocha Dourado, M.; de Oliveira Pires, M. B.; Pêgo, S. P. B. \& de Freitas, E. M. Palate hyperpigmentation caused by prolonged use of the anti-malarial chloroquine. Head Neck Pathol., 6(1):48-50, 2012.

Dziedzic, A. \& Wojtyczka, R. The impact of coronavirus infectious disease 19 (COVID-19) on oral health. Oral Dis., 2020. DOI: https://www.doi.org/10.1111/odi.13359

European Centre for Disease Prevention and Control. Coronavirus disease 2019 (COVID-19) pandemic: increased transmission in the EU/EEA and the UK - seventh update, 25 March 2020. Estocolmo, European Centre for Disease Prevention and Control, 2020. Disponible en: https://www.ecdc.europa.eu/sites/ default/files/documents/RRA-seventh-update-Outbreak-ofcoronavirus-disease-COVID-19.pdf

Godinho, G. V.; Paz, A. L. L. M.; de Araújo Gomes, E. P. A.; Garcia, C. L. \& Volpato, L. E. R. Extensive hard palate hyperpigmentation associated with chloroquine use. Br. J. Clin. Pharmacol., 2020. DOI: https://www.doi.org/10.1111/bcp.14313

Imsand, M.; Janssens, J. P.; Auckenthaler, R.; Mojon, P. \& BudtzJørgensen, E. Bronchopneumonia and oral health in hospitalized older patients. A pilot study. Gerodontology, 19(2):66-72, 2002. 
Jimenez-Cauhe, J.; Ortega-Quijano, D.; Carretero-Barrio, I.; SuarezValle, A.; Saceda-Corralo, D.; Moreno-Garcia del Real, C. \& Fernandez-Nieto, D. Erythema multiforme-like eruption in patients with COVID-19 infection: clinical and histological findings. Clin. Exp. Dermatol., 2020. DOI: https://www.doi.org/10.1111/ ced.14281

Lechien, J. R.; Chiesa-Estomba, C. M.; De Siati, D. R.; Horoi, M.; Le Bon, S. D.; Rodriguez, A.; Dequanter, D.; Blecic, S.; El Afia, F.; Distinguin, L.; et al. Olfactory and gustatory dysfunctions as a clinical presentation of mild-to-moderate formsof the coronavirus disease (COVID-19): a multicenter European study. Eur. Arch. Otorhinolaryngol., 277(8):2251-61, 2020.

Li, Q.; Guan, X.; Wu, P.; Wang, X.; Zhou, L.; Tong, Y.; Ren, R.; Leung, K. S. M.; Lau, E. H. Y.; Wong, J. Y.; et al. Early transmission dynamics in Wuhan, China, of novel coronavirus-infected pneumonia. N. Engl. J. Med., 382(13):1199-207, 2020.

Melián-Rivas, A.; Calcumil-Herrera, P.; Boin-Bakit, C. \& CarrascoSoto, R. Detection of COVID-19 (SARS-CoV-2) by saliva: a lowinvasive diagnostic alternative. Int. J. Odontostomat., 14(3):31620, 2020.

Menni, C.; Valdes, A.; Freydin, M. B.; Ganesh, S.; Moustafa, J. E. S.; Visconti, A.; Hysi, P.; Bowyer, R. C. E.; Mangino, M.; Falchi, $\mathrm{M}$; et al. Loss of smell and taste in combination with other symptoms is a strong predictor of COVID-19 infection. MedRxiv, 2020. DOI: https://www.doi.org/10.1101/2020.04.05.20048421

Parma, V.; Ohla, K.; Veldhuizen, M.; Niv, M. Y.; Kelly, C. E.; Bakke, A. J.; Cooper, K. W.; Bouysset, C.; Pirastu, N.; Dibattista, M.; et al. More than smell. COVID-19 is associated with severe impairment of smell, taste, and chemesthesis. MedRxiv, 2020. DOI: https://www.doi.org/10.1101/2020.05.04.20090902

Pedrosa, M. S.; Sipert, C. R. \& Nogueira, F. N. Salivary glands, saliva and oral findings in COVID-19 infection. Pesqui. Bras. Odontopediatria Clin. Integr., 20(Supl. 1):e0104, 2020.

Peng, X.; Xu, X.; Li, Y.; Cheng, L.; Zhou, X. \& Ren, B. Transmission routes of 2019-nCoV and controls in dental practice. Int. J. Oral Sci., 12(1):9, 2020.

Putra, B. E.; Adiarto, S.; Dewayanti, S. R. \& Juzar, D. A. Viral exanthem with "Spins and needles sensation" on extremities of a COVID-19 patient: A self-reported case from an Indonesian medical frontliner. Int. J. Infect. Dis., 96:355-8, 2020.

Quagliarello, V.; Ginter, S.; Han, L.; Van Ness, P.; Allore, H. \& Tinetti, M. Modifiable risk factors for nursing home-acquired pneumonia. Clin. Infect. Dis., 40(1):1-6, 2005.

Salehi, M.; Ahmadikia, K.; Mahmoudi, S.; Kalantari, S.; Jamalimoghadamsiahkali, S.; Izadi, A.; Kord, M.; Manshadi, S. A. D.; Seifi, A.; Ghiasvand, F.; et al. Oropharyngeal candidiasis in hospitalised COVID-19 patients from Iran: Species identification and antifungal susceptibility pattern. Mycoses, 63(8):771-8, 2020.

Sampson, V.; Kamona, N. \& Sampson, A. Could there be a link between oral hygiene and the severity of SARS-CoV-2 infections? Br. Dent. J., 228:971-5, 2020.

Scully, C. \& Samaranayake, L. P. Emerging and changing viral diseases in the new millennium. Oral Dis., 22(3):171-9, 2016.

Sepúlveda, C. V.; Waissbluth, A. S. \& González, G. C. Anosmia y enfermedad por Coronavirus 2019 (COVID-19): ¿Qué debemos saber?. Rev. Otorrinolaringol. Cir. Cabeza Cuello, 80(2):247-58, 2020.

Soares, C. D.; de Carvalho, R. A.; de Carvalho, K. A.; de Carvalho, M. G. F. \& de Almeida, O. P. Letter to Editor: Oral lesions in a patient with Covid-19. Med. Oral Patol. Oral Cir. Bucal, 25(4):e563-4, 2020.

Suzuki, A. \& Iwata, J. Molecular regulatory mechanism of exocytosis in the salivary glands. Int. J. Mol. Sci., 19(10):3208, 2018.

Trayes, K. P.; Love, G. \& Studdiford, J. S. Erythema multiforme: recognition and management. Am. Fam. Physician, 100(2):828, 2019.
World Health Organization (WHO). Coronavirus disease (COVID19) Situation Report-191, 2020. Ginebra, World Health Organization, 2020. Disponible en: https://www.who.int/docs/ default-source/coronaviruse/situation-reports/20200729-covid19-sitrep-191.pdf?sfvrsn=2c327e9e_2

Xu, H.; Zhong, L.; Deng, J.; Peng, J.; Dan, H.; Zeng, X.; Li, T. \& Chen, Q. High expression of ACE2 receptor of 2019-nCoV on the epithelial cells of oral mucosa. Int. J. Oral Sci., 12(1):8, 2020.

Yan, C. H.; Faraji, F.; Prajapati, D. P.; Ostrander, B. T. \& DeConde, A. S. Self-reported olfactory loss associates with outpatient clinical course in COVID-19. Int. Forum Allergy Rhinol., 10(7):82131, 2020.

Dirección para correspondencia:

María Eugenia Nemeth Kohanszky

Los Olivillos 451, Chiguayante

Concepción

CHILE

Correo: marnemethk@udd.cl

Recibido : 30-07-2020

Aceptado: 06-08-2020 